\title{
Salas de Lactancia para Instituciones Públicas o Privadas y Empresas
}

\section{Lactation rooms for Public or Private Institutions and Corporations}

\author{
Ministerio de Salud Pública y Bienestar Social
}

\section{INTRODUCCIÓN}

Amamantar es mucho más que nutrir al niño/a. Es un proceso que involucra la interacción profunda entre la madre y el hijo, con repercusiones en el estado nutricional del niño/a, en su habilidad de defenderse de infecciones, en su fisiología y en su desarrollo cognitivo y emocional, además de tener implicancias en la salud física y síquica de la madre ${ }^{(1)}$.

A pesar de todas las evidencias científicas que demuestran la superioridad de la lactancia sobre otras formas de alimentar a los niños y niñas pequeños, y a pesar de los esfuerzos de los diversos organismos nacionales e internacionales, las tasas de lactancia materna en Paraguay, en especial la lactancia materna exclusiva están bastante por debajo de lo recomendado ${ }^{(2)}$.

En nuestro país, la segunda causa para que las madres dejen de amamantar lo constituye el retorno al trabajo o al estudio ${ }^{(2)}$.

En este sentido, el entorno institucional/laboral debe reconocer a la mujer como protagonista de su proceso de amamantamiento, valorizándola, escuchándola, empoderándola y creando las condiciones necesarias para promover, proteger y apoyar de manera a lo lograr una lactancia exitosa que redundará en beneficios tanto para el bebé, la madre y por consiguiente a la familia.

\section{JUSTIFICACIÓN}

\section{Recomendaciones del Ministerio de Salud Pública}

El Ministerio de Salud Pública y Bienestar Social como ente rector de la salud en el Paraguay ratifica las recomendaciones de la Organización Mundial de la Salud que declara en su Estrategia Mundial para la Alimentación del Lactante y del Niño Pequeño ${ }^{(3)}$ : “La lactancia natural es una forma sin paragón de proporcionar un alimento ideal para el crecimiento y el desarrollo sano de los lactantes; también es parte integrante del proceso reproductivo, con repercusiones importantes en la salud de las madres. Como recomendación de salud pública mundial, durante los seis primeros meses de vida los lactantes deberían ser alimentados exclusivamente con leche materna para logra un crecimiento, un desarrollo y una salud óptimos ${ }^{(4)}$. A partir de ese momento, a fin de satisfacer sus requisitos nutricionales en evolución, los lactantes deberían recibir alimentos complementarios adecuados e inocuos desde el punto de vista nutricional, sin abandonar la lactancia natural por lo menos hasta los 2 años de edad, o más tarde. La lactancia natural exclusiva debe practicarse desde el nacimiento, salvo el caso de algunas afecciones médicas relacionadas a la madre o al bebé, y si se practica sin limitaciones, propicia una abundante producción de leche". Y agrega. "Las madres también deberían poder seguir amamantando y cuidando a sus hijos al retomar a su empleo remunerado, lo que se puede lograr aplicando medidas legislativas y otras medidas conexas compatibles con el Convenio de la

Lineamientos para la habilitación de Salas de Lactancia en Instituciones y Empresas públicas y privadas, aprobada por Resolución S. G. № 396, Ministerio de Salud Pública y Bienestar Social 
OIT sobre la protección de la maternidad, 2000, № 183, y la Recomendación sobre la protección de la maternidad, 2000, № 191. Todas las mujeres empleadas fuera del hogar deberían poder disponer de una licencia de maternidad, de guarderías y de pausas remuneradas para amamantar".

Siguiendo las recomendaciones internacionales, la legislación Nacional contempla en el art 89 de la Constitución Nacional y art. 133-134 del Código Laboral medidas de protección para la mujer embarazada y en periodo de lactancia.

\section{Un empresario/empleador que facilita a sus empleadas el amamantar, logra:}

* Mejor satisfacción de sus empleadas/os: Al poder combinar el trabajo con la maternidad - en este caso expresada por la continuación de la lactancia materna-, sus empleadas y empleados se sentirán satisfecho pero además reconocidos, respetados y apoyados en su papel de madres y padres ${ }^{(5)}$.

* Mayor fidelidad de las mujeres trabajadoras para con su empresa, como gratitud a su colaboración y sensibilidad: Esa satisfacción y ese reconocimiento a su colaboración y sensibilidad volverán en forma de fidelidad e identificación para con la empresa ${ }^{(6)}$.

* Menor ausentismo, ya que esos bebés y sus madres enfermarán menos: Las madres cuyos niños reciben alimentación con fórmulas se ausentan (para cuidar a sus hijos enfermos) más del doble de días que quienes amamantan ${ }^{(7)}$. La compañía de seguros CIGNA llevó a cabo un estudio de 2 años sobre 343 empleadas que participaron en su programa de apoyo a la lactancia, y encontró que el programa tuvo como resultado un ahorro de $\$ 60.000$ por reducción del ausentismo ${ }^{(8)}$.

* Mayor tasa de rápido regreso al trabajo: Las empleadas tienen más probabilidades de volver al trabajo al finalizar su licencia por maternidad (esto es sin tomar licencias extraordinarias), cuando su lugar de trabajo ofrece un entorno propicio para la lactancia materna ${ }^{(6)}$.

* Mayor rendimiento de las horas trabajadas: Ya que al tener un hijo sano la madre trabajadora no debe ocupar su pensamiento en preocupaciones y angustias.

* Disminución de la rotación o pérdida de personal calificado y aún su pérdida a causa de nacimiento de un bebé: Con el consiguiente ahorro en reclutamiento y capacitación de nuevo personal, además del tiempo necesario para su rendimiento óptimo ${ }^{(6)}$.

* Mejorar la imagen corporativa: Al ocuparse del bienestar de las mujeres trabajadoras y sus familias, mostrando cómo la responsabilidad social comienza por la propia casa.

* Menor costo de atención de la salud: Un estudio encontró que por cada 1.000 bebés no amamantados, hubo 2.033 consultas médicas adicionales, 212 días de hospitalización adicional, y 609 recetas médicas adicionales solo para 3 enfermedades - oído, respiratorias e infecciones gastrointestinales ${ }^{(9)}$. Esto no incluyó los riegos de muchas otras enfermedades de la infancia, o enfermedades de la mujer como el cáncer de mama pre-menopáusico, que se reducen cuando una madre amamanta ${ }^{(10)}$.

* Favorecimiento de una fuerza laboral más saludable para el futuro: El impacto de la lactancia materna sobre el bebé se prolonga hasta la vida adulta, previniendo obesidad, diabetes, hipertensión y algunos tipos de cáncer ${ }^{(11)}$.

\section{Beneficios para la madre trabajadora y su niño/a}

* La lactancia materna ofrece una experiencia única de salud y genera armonía en la relación madre-bebé.

* Desde el punto de vista nutricional, no existe una real alternativa a la lactancia materna; las leches artificiales son productos en general de origen vacuno, modificado y carente de muchas sustancias inmunológicas.

* Desde el punto de vista inmune, solo la leche materna puede brindarle al bebé los anticuerpos que todavía no es capaz de generar por sí mismo; por lo tanto, enfermará mucho menos, y menos importantes serán sus problemas de salud ${ }^{(11)}$.

* Desde el punto de vista de la madre, la lactancia materna previene diversas enfermedades de la mujer, como cierto tipo de cáncer. Además, es la forma natural de perder los kilos que el embarazo deja, precisamente, para la producción de leche en los primeros meses de vida ${ }^{(11)}$.

* Desde el punto de vista emocional, las madres 
que sostienen la lactancia cuando retornan a su trabajo sienten menos culpa o sensación de estar abandonando a sus bebés.

\section{OBJETIVO}

Establecer las condiciones adecuadas para que las madres estudiantes o trabajadoras en periodo de lactancia a su regreso al trabajo/estudio encuentren un lugar amigable, cálido e higiénico donde puedan extraer y conservar la leche materna bajo normas técnicas de seguridad, para luego transportarla al hogar y ofrecerla al bebé en aquellos momentos que no pueden estar juntos.

\section{LINEAMIENTOS PARA SALAS DE LACTANCIA}

1. Normativa de lactancia materna en las instituciones y empresas:

* La Institución/Empresa promueve, protege y apoya la lactancia materna de las mujeres en periodo de amamantamiento de su empresa/institución.

* Mientras dure el periodo de lactancia, especialmente durante los primeros 6 meses de vida, la Institución/Empresa brindará las condiciones para apoyar la lactancia materna exclusiva, luego de ese periodo, continuará ofreciendo apoyo para mantener la lactancia materna conforme a las recomendaciones de OPS/OMS.

* Para los efectos arriba señalados será flexible respecto a las pausas para lactancia establecida en el Código Laboral (Ley 213/93). Teniendo en cuenta el tiempo establecido en una jornada laboral, la mujer necesita unos 15 a 20 minutos para extraerse la leche cada vez para mantener una buena producción de leche.

* La Institución/Empresa dispondrá de un espacio destinado a la extracción de la leche materna que denominaremos SALA de LACTANCIAMATERNA, este lugar deberá ser:

* Higiénico;

* Privado;

* Confortable;

* Tranquilo; y

* Fácilmente accesible.

* La Institución/Empresa deberá garantizar el aseo del área así como la provisión de los insumos necesarios (jabón líquido con dispensador para el lavado de manos, toallas de papel, recipientes medianos con tapa y pedal para la basura, y percheros).
* La Institución/Empresa implementará un Sistema de Registro de utilización de la Sala de Lactancia.

\section{Requisitos para sala de lactancia:}

* Sala de dimensiones adecuadas a la cantidad de mujeres que puedan requerir de ella en forma simultánea, con elementos de confort e intimidad para que cada mujer pueda realizar la extracción de su propia leche de manera privada, cómoda y absolutamente higiénica.

* Cada unidad deberá contar con al menos una silla cómoda, un lavamanos con agua fría/caliente, jabón líquido y toallas de papel, un refrigerador con termómetro para control diario de temperatura, de uso exclusivo para el fin de la sala de lactancia.

* Una unidad debería tener una superficie mínima de 2 metros x 2 metros (para un sillón), más 1,5 m² por cada sillón extra instalado.

* Excelente iluminación (de ser posible natural), ventilación y decoración alegre; ambiente climatizado.

* El o los lavatorios no deben ser compartidos con baños u otras instalaciones sanitarias del edificio.

* Tomacorriente eléctrico (para el uso de una bomba mecánica, si se desea).

* Una pizarra o panel donde las mujeres que usan la sala puedan colocar fotos de sus bebés para mirarlas al mismo tiempo que extraen su leche para favorecer la "bajada" de la leche.

* Adicionalmente, se recomienda dotar a la Sala de Lactancia con material educativo relacionado con el tema, como cartillas, afiches o dípticos y si es posible música.

* Disponibilidad, por parte de la institución o de las propias estudiantes o trabajadoras, de envases aptos para la recolección y almacenamiento (frascos de vidrio con tapa de plástico de boca ancha: 60, 90 y 180 $\mathrm{ml}$; cinta de enmascarar y marcador para rotular los frascos) y recipientes térmicos para su transporte.

* En la mayoría de instituciones/empresas existe un departamento médico, con un profesional en salud que puede ser capacitado para informar y aconsejar 
a las madres sobre distintos aspectos de la lactancia materna, así como para dar el apoyo que permita solucionar problemas que pudieran dificultar la lactancia y la extracción de leche materna.

\section{Otras medidas para apoyar la lactancia en el lugar de trabajo}

* Facilite la entrega de folletos e instructivos de promoción de lactancia materna.

* Posibilite que sus empleadas retornen gradualmente al trabajo o facilite horarios flexibles a fin de permitirles adaptarse a la separación con sus bebés.

* Autorice que en determinadas tareas que así lo permitan su personal pueda estar, si lo desea, acompañada de su bebé.

* Si le es posible provea de los insumos necesarios (frascos estériles, contenedores térmicos de transporte) para facilitar la tarea de las madres.

* Cuando le sea posible, favorezca la ejecución de tareas a distancia, por ejemplo desde la propia casa de su empleada, o medias jornadas de trabajo.

* Favorezca la participación de los padres en el nacimiento y días posteriores mediante licencias por paternidad adecuadas.

* Permita que la madre pueda retirarse unos minutos del trabajo para amamantar al bebé en algún sitio cercano.

\section{ANEXOS}

\section{Lavado de Manos}

\section{¿Cómo lavarse las manos?}

¡Lávese las manos solo cuando estén visiblemente sucias! Si no, utilice la solución alcohólica

(c)

0

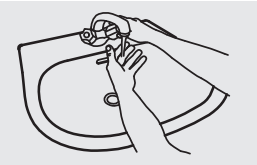

Mójese las manos con agua;

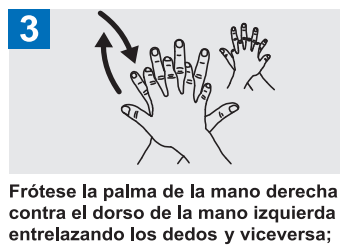
entrelazando los dedos y viceversa;

6

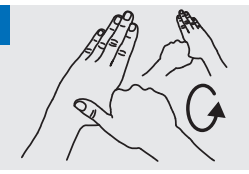

Frótese con un movimiento de rotación el pulgar izquierdo, atrapándolo con la palma de la mano derecha y viceversa;

9

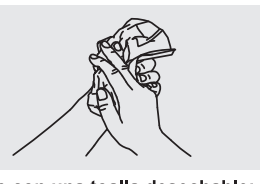

Séquese con una toalla desechable;

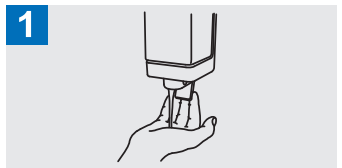

Deposite en la palma de la mano una cantidad de jabón suficiente para cubrir todas las superficies de las manos:

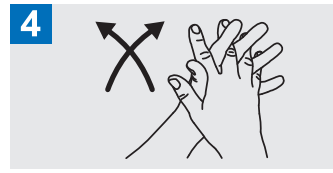

Frótese las palmas de las manos entre sí, con los dedos entrelazados:

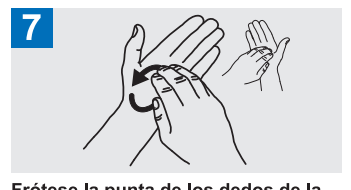

Frótese la punta de los dedos de la mano derecha contra la palma de movimiento de rotación y viceversa;
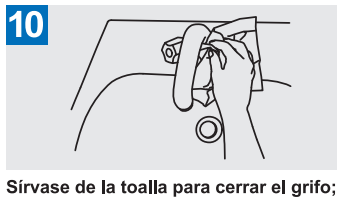

Seguridad del Paciente
2

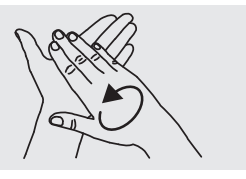

Frótese las palmas de las manos entre sí;

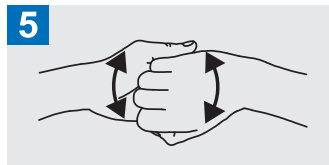

Frótese el dorso de los dedos de una mano con la palma de la mano opuesta, agarrándose los dedos;

8

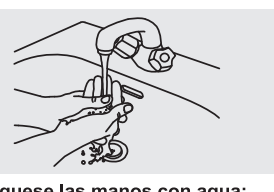

Enjuáguese las manos con agua;

11

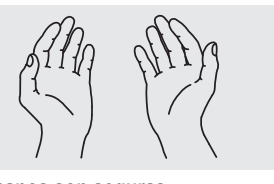

Sus manos son seguras.

\section{SAVE LIVES}

Clean Your Hands 


\section{Extracción manual de leche y conservación}

\section{Extracción manual de la Leche Materna}

Para que tu bebé se alimente mientras estás fuera de casa o para aliviar la congestión del pecho, podés sacar tu leche de la siguiente forma:

1. Preparación: lávate las manos (ver anexo 1).

2. Estimulación: presiona firme y cuidadosamente tu pecho hacia el tórax y realiza movimientos circulares con los dedos en diferentes puntos desde la base de la mama hacia el pezón.

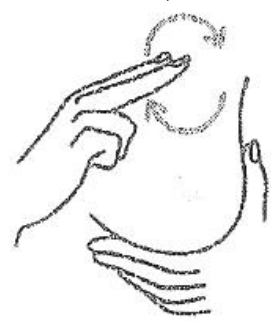

3. Extracción: con el pulgar por arriba y los índice y medio por abajo del pezón con la areola entre ellos, empuja tu pecho hacia las costillas y mediante movimientos giratorios hacia el pezón, presiona y vacía la mama suavemente sin deslizar los dedos.
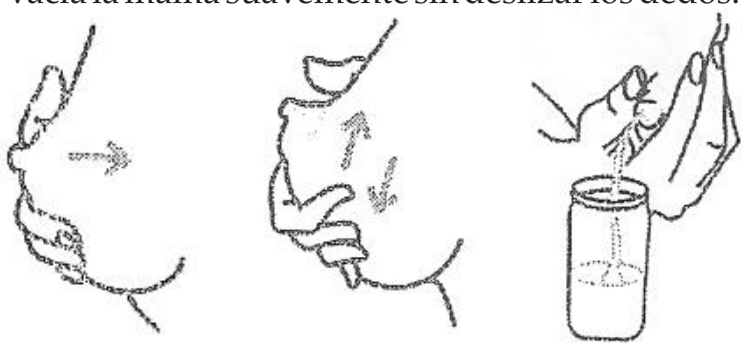

\section{CONSERVACIÓN}

Una vez extraída la leche se almacenará en recipientes limpios, y preferiblemente estériles. Es conveniente usar un recipiente limpio cada vez que se recolecte leche y etiquetar la leche con la fecha y la hora en que se extrajo.

Las mejores opciones para almacenar leche humana son recipientes de vidrio o plástico duro con tapas que cierren bien. Ante la controversia sobre el efecto del policarbonado PPD (bisphenol A=BPA) en la salud no se debe usar recipientes que los contenga.

La leche materna se conserva intacta por 2 horas a temperatura ambiente, en heladera durante 24 a 48 hs, y en congelador de la heladera durante 2 semanas.

Es recomendable distribuir la leche en envases y porciones iguales a lo que el niño consume cada vez.

\section{Recursos adicionales y sitios web}

* Organización Panamericana de la Salud: www.paho.org/alimentacioninfantil

* Organización Internacional del Trabajo: www.ilo. org/global/lang--es/index.htm

* Red Mundial de Grupos pro Alimentación Infantil (IBFAN): www.ibfan-alc.org

* World Alliance Breastfeeding Action: www.waba.org

* Liga de la Leche: www.Illi.org

* Academia Americana de Pediatría: www.aap.org

* Business case for breastfeeding: www.cdph.ca. gov/HealthInfo/healthyliving/childfamily/Pages/B usiness-CaseforBreastfeeding.aspx

* Ver también: Lactancia y trabajo: Folleto para los compañeros de trabajo y Lactancia y trabajo: Folleto para madres y futuras madres. (OPS, 2011) y disponible en www.paho.org/alimentacioninfantil. Publicado: diciembre, 2011.

\section{Resolución S. G. № 396 del Ministerio de Salud Pública y Bienestar Social}

RESOLUCIÓN S. G. № 396 POR LA CUAL SE APRUEBAN LOS LINEAMIENTOS PARA LA HABILITACIÓN DE SALAS DE LACTANCIA EN INSTITUCIONES Y EMPRESAS PÚBLICAS $Y$ PRIVADAS

Asunción, 5 de agosto de 2014.

\section{VISTO:}

La recomendación de la Organización Mundial de la Salud (OMS) sobre los beneficios de la lactancia materna; el llamado para apoyar a las madres en periodo de lactancia; y la normativa nacional e internacional vigente en la materia; $y$

\section{CONSIDERANDO:}

Que la Constitución Nacional, en el artículo 68, DEL DERECHO A LA SALUD establece: “El Estado 
protegerá y promoverá la salud como derecho fundamental de la persona y en interés de la comunidad"; así también, garantiza la protección de la mujer en periodo de maternidad, específicamente en el artículo 89.- DEL TRABAJO DE LAS MUJERES, que dispone: "Los trabajadores de uno y otro sexo tienen los mismos derechos y obligaciones laborales, pero la maternidad será objeto de especial protección, que comprenderá los servicios asistenciales y los descansos correspondientes, los cuales no serán inferiores a doce semanas...".

Que la Convención de los Derechos del Niño, acogida por Ley 57/90, con rango constitucional, dispone en el artículo 24, inc. "e) Asegurar que todos los sectores de la sociedad, y en particular los padres y los niños, conozcan los principios básicos de la salud y la nutrición de los niños, las ventajas de la lactancia materna... tengan acceso a la educación pertinente y reciban apoyo en la aplicación de esos conocimientos...".

Que el Código Laboral, art. 134, dice: "En el período de lactancia, las madres trabajadoras tendrán dos descansos extraordinarios por día, de media hora cada uno, para amamantar a sus hijos. Dichos descansos serán considerados como períodos trabajados, con goce de salarios. A este fin, los establecimientos industriales o comerciales en que trabajen más de cincuenta mujeres, están obligados a habilitar salas maternales para niños menores de dos años, donde éstos quedarán bajo custodia, durante el tiempo de ocupación de las madres. Esta obligación cesará cuando las instituciones de seguridad social atiendan dicha asistencial".

Que el Código de la Niñez y la Adolescencia, en el artículo 10, DE LA RESPONSABILIDAD DEL ESTADO, estable: "a) atender a la mujer embarazada insolvente, a la que se proveerá de alojamiento, alimentación y medicamentos necesarios; $b$ ) atender a la embarazada indígena, en el marco del más amplio respeto a su cultura; c) elaborar planes de atención especializada para la protección de la adolescente embarazada; $y, d$ ) promover la lactancia materna".

Que la Ley № 836, Código Sanitario, dispone en el artículo 3 que: "El Ministerio de Salud Pública y Bienestar Social es la más alta dependencia del Estado competente en materia de salud y aspectos fundamentales del bienestar social".

Que la Ley № 836/80, Código Sanitario, establece en el Artículo 5: "La política nacional de salud y bienestar social será establecida, periódicamente por el Poder Ejecutivo"; en el Artículo 6o: "Los planes y programas de salud y bienestar social deberán elaborarse de acuerdo con los objetivos, políticas y estrategias globales del desarrollo económico y social de la Nación", y en el Artículo 7ㅇ: "Los planes, programas y actividades de salud y bienestar social, a cargo de las instituciones públicas y privadas, serán aprobadas y controladas por el Ministerio que debe orientarlos de acuerdo con la política de salud y bienestar social de la Nación".

Que en el artículo $14^{\circ}$ de la Ley № $836 / 80$ dispone: "La salud del grupo familiar es derecho irrenunciable que se reconoce a todos los habitantes del país. El Estado promoverá y realizará las acciones necesarias en favor de la salud familiar".

Que el artículo 286ำ establece: El Ministerio promoverá la responsabilidad familiar, fundamentalmente en el desarrollo y educación del menor. Estimulará la creación de hogares sustitutivos para los menores desamparados y coordinará con las actividades de otras entidades, públicas o privadas, en la materia".

Que, como ente rector en materia de salud pública, el Ministerio de Salud Pública y Bienestar Social acoge las recomendaciones de la Organización Mundial de la Salud (OMS) recogidas en la Estrategia Mundial para la Alimentación del Lactante y del Niño Pequeño, que dice: "La lactancia natural es una forma sin paragón de proporcionar un alimento ideal para el crecimiento y el desarrollo sano de los lactantes...".

Como recomendación de salud pública mundial, durante los seis primeros meses de vida, los lactantes deberían ser alimentados exclusivamente con leche materna para lograr un crecimiento, un desarrollo y una salud óptimos. A partir de ese momento, con el fin de satisfacer sus requisitos nutricionales en evolución, los lactantes deberían recibir alimentos complementarios adecuados e inocuos desde el punto de vista nutricional, sin abandonar la lactancia natural por lo menos hasta los 2 años de edad, o más tarde. La lactancia natural exclusiva debe practicarse desde el nacimiento, salvo el caso de algunas afecciones médicas relacionadas a la madre o al bebé, y si se practica sin limitaciones, propicia una abundante producción de leche. Y agrega: Las madres también deberían poder seguir amamantando y cuidando a sus hijos al retomar a su empleo remunerado, lo que se puede lograr aplicando medidas legislativas y otras 
medidas conexas compatibles con el Convenio de la OIT sobre la protección de la maternidad, 2000, № 183, y la Recomendación sobre la protección de la maternidad, 2000, №191. Todas las mujeres empleadas fuera del hogar deberían poder disponer de una licencia de maternidad, de guarderías y de pausas remuneradas para amamantar.

Que, teniendo en consideración las disposiciones antes citadas, por las cuales se establece y garantiza el derecho de la mujer a recibir apoyo durante la maternidad y el periodo de lactancia, y se reconocen los beneficios de la lactancia materna para la madre, el niño y la salud pública, se ve la necesidad de brindar condiciones óptimas a la madre y el niño en esta etapa. En consecuencia, el Ministerio de Salud Pública y Bienestar Social, como órgano responsable de dictar la política nacional de salud, impulsa la instalación de salas de lactancia en las instituciones y empresas públicas y privadas como medida necesaria para alentar a las madres a seguir amamantando una vez que se incorporan a la vida laboral y ve necesario contar con lineamientos que establezcan las condiciones adecuadas para que las madres estudiantes o trabajadoras en periodo de lactancia, a su regreso al trabajo/estudio encuentren un lugar amigable, cálido e higiénico donde puedan extraer y conservar la leche materna bajo normas técnicas de seguridad, para luego transportarla al hogar y ofrecerla al bebé en aquellos momentos en que no pueden estar juntos.

Que el Decreto 21.376/98 en el Artículo 4ํㅜㄴ dispone: "El derecho de todas las personas a un nivel adecuado y digno de vida en lo que respecta a la salud y al bienestar, no solo descansa en la atención médica y sanitaria, sino que comprende a la vez al derecho a un nivel adecuado de alimentos, agua, vivienda, educación, trabajo y servicios sociales desde una perspectiva de género y dentro de un marco de libertad, paz, seguridad y equidad, en consonancia con un ambiente saludable", en el Artículo 9o, numeral 5o: "Son funciones específicas en el área de la Salud y del Ambiente asegurar la promoción y la protección de la salud de la población, como funciones esenciales de la salud pública y responsabilidad del Estado", en el Artículo 13', numeral 3: "Son funciones específicas en el área de Bienestar Social diseñar, coordinar y ejecutar planes, programas y proyectos de promoción, prevención y protección, en base a una red de servicios sociales, comunitarios, intermedios, especializados y otras modalidades de apoyo para la Familia, la Mujer, la Infancia, la Juventud y los Adultos Mayores, a nivel nacional, regional y local", y en el Artículo 20ㅜ, numeral 1, "Son funciones específicas del Ministerio de Salud Pública y Bienestar Social determinar los objetivos y desarrollo de la Política de Salud Humana y Ambiental y de Bienestar Social".

Que el Decreto 21.376/98 en su Artículo 20, numeral 7, establece que una de las funciones específicas del Ministerio es la de dictar Resoluciones que regulen la actividad de los diversos programas y servicios, reglamenten su organización y determinen sus funciones;

POR TANTO, en ejercicio de sus atribuciones legales;

\section{EL MINISTRO DE SALUD PÚBLICA Y BIENESTAR SOCIAL}

\section{RESUELVE:}

Artículo 10. Aprobar los lineamientos para la habilitación de salas de lactancia en instituciones y empresas públicas y privadas.

Artículo 2º. Encomendar el control, habilitación y registro de salas de lactancia a la Dirección de Establecimientos de Salud, Afines y Tecnología Sanitaria, dependiente de la Dirección General de Vigilancia de Salud, conforme a los lineamientos aprobados en el artículo anterior.

Artículo 3\%. Comunicar a quienes corresponda y cumplido, archivar.

DR. ANTONIO CARLOS BARRIOS F. MINISTRO 


\section{REFERENCIAS}

1. Cadernos de Atenção Básica no 23, Saúde da Criança: Nutrição Infantil. Ministério da Saúde, 2009.

\section{CEPEP 2008.}

3.Disponible en http://www.who.int/nutrition/publica tions/gs_infant_feeding_text_spa.pdf

4. Según se formula en las conclusiones y recomendaciones en la reunión consultiva de expertos (Ginebra, 28 a 30 de marzo de 2001 que ultimó el examen sistemático de la duración óptima de la lactancia natural exclusiva (véase el documento A54/INF.DOC./4). Véase también la resolución WHA54.2.

5. Galtry J. (1997). Lactation and the labor market: Healthcare Research and Quality (2007). Breastfeeding and breastfeeding, labor market changes, and public policy in the maternal and infant health outcomes in developed countries. United States. Health Care Women Int., 18, 467-480.

6. U.S. Department of Health and Human Services. Health Resources and Services Admistration. The Business Case for Breastfeeding. Steps for Creating a Breastfeeding Friendly Worksite. Disponible en: http://www.ask.hrsa. gov/detail_material.cfm?ProdID=4135. Última consulta: 20/07/2011.
7. Cohen R, Mrtek MB \& Mrtek RG. (1995). Comparison of maternal absenteeism and infant illness rates among breastfeeding and formula-feeding women in two corporations. American Journal of Health Promotion, 10(2), 148-153.

8. Dickson V, Hawkes C, Slusser W, Lange L, \& Cohen R. (2000). The positive impact of a corporate lactation program on breastfeeding initiation and duration rates: help for the working mother. Unpublished manuscript. Presented at the Annual Seminar for Physicians, cosponsored by the American International, on July 21, 2000.

9. Ball T \& Wright A. (1999). Health care costs of formulafeeding in the first year of life. Pediatrícs, 103 (4), 871-876.

10. U.S. Department of Health and Human Services, Agency for Healthcare Research and Quality (2007). Breastfeeding and maternal and infant health outcomes in developed countries. Evicence report, Technology Assessment, Number 153.9.

11. León-Cava, Natalia. Cuantificación de los beneficios de la Lactancia Materna: Reseña de la Evidencia. Washington, D.C.: OPS, 2002. Disponible en http://paho. org/spanish/ad/fch/BOB-Main.htm (última consulta 20/07/2011). 